\title{
Network Modeling and Visualization Platform based on Moodle and Mxgraph
}

\author{
Fang-jian He, Xiao-pan Zhang, Xiao-yan Ma \\ School of Resources and Environmental Engineering, Wuhan University of Technology, Wuhan, Chin
}

\begin{abstract}
For the model of learning, Most of the information of relevant professional beginners are difficult to adapt to the training of the model structure. In the early learning students should focus on the cultivation of thinking structure, visual modeling study is conducive to the strengthening of the relevant professional structured learning and understanding. Nowadays Study on the visualization of teaching are few all over the world, Visualization technology is often manifested as data visualization, but the visualization model of image network still remain at the theoretical level. In order to meet the requirements of teaching at present, we design and build the network platform of visual modeling training. Take advantage of the network script language, Moodle and Mxgraph, which have the correlation of modelling, the platform is developed in the server environment. The modeling steps platform record students, in order to guide the development of structured programming thinking, to help the designer to master and practice model.
\end{abstract} platform

Index Terms - Modeling and visualization, structured, network

In the teaching process, we should strengthen students' understanding of the model[1]. In the absence of visual teaching aid. The main research field of visualization is the visualization of teaching of computer technology, where image express some abstract problems intuitively in Teaching, it is proposed the establishment of visual model in the teaching and training platform services. At the same time the single teaching method makes students lack of clarity on the teaching requirements, which did not issue a structured way of thinking to think. Using modeling ideology teaching visualization helps to stimulate students' interest and understand the course content better. The main research field of visualization is the visualization of teaching of computer technology, where image express some abstract problems Intuitively in Teaching, it is proposed the establishment of visual model in the teaching and training platform services. Gene expression is the idea of the model, so implementation model visualization has important practical significance for modern teaching. Teaching visualization helps teachers improve teaching efficiency, which helps students understand what they have learned to master the curriculum.

\section{The Demand of Modeling and Visualization Platform}

Visual thinking teaching appears in recent years, using visual tool for mining the potential of students' memory and imagination, Improving students' way of thinking, enhancing the efficiency of teaching research. Teaching modeling visualization is enhanced teaching and research of great significance, mainly for students to deepen their intuitive understanding of some models by rendering, but also to learn how to use the model to solve the education problem.

Combined with a variety of information, Modeling visualization can be summarized as abstract and difficult to describe things or relationships through a number of related data processing to make it intuitive, visualized. In the research on visualization modeling teaching, compared with the traditional teaching can be found that combined with the model of teaching can enable students to understand the teaching task. The students themselves establish the model, which also converts structure of thinking into the process of space structure, showing their reachability and topology. For example, in the programming process of learning, Visual training adds algorithms and modeling of two modules, letting beginners only need to draw the flow chart for the learning algorithm.

\section{The Network Framework of Model's Visual Platform}

The rapid development of the technology of model's visualization is based on the visualization technology of graphics, besides, visualization is the theory, method and technology of interactive processing by computer. Due to the high requirements of image, the teaching model is bound to get rid of the traditional teaching methods. Combining with the requirements of teaching curriculum, it is prevalent to establish teaching platforms which support interactive information. Visual learning need to display in the WEB platform. At the same time, as for the teaching management, the visual learn of WEB could provide the network teaching platform, which is convenient for evaluation, communication and monitoring of study. Besides, the popularity of the network makes the students can realize remote visualization modeling training in any place .

As for as the establishment of the platform, we should consider that the important curriculum and experiment related professional integrated into this platform and Setting up several different professional courses on he platform.

In view of the above content, we divide the whole system into six different levels of the following: curriculum management modular, task management modular, user course selection authentication modular, model establishment modular, data monitor and interact modular, structure query modular, frame as shown in Fig1. 


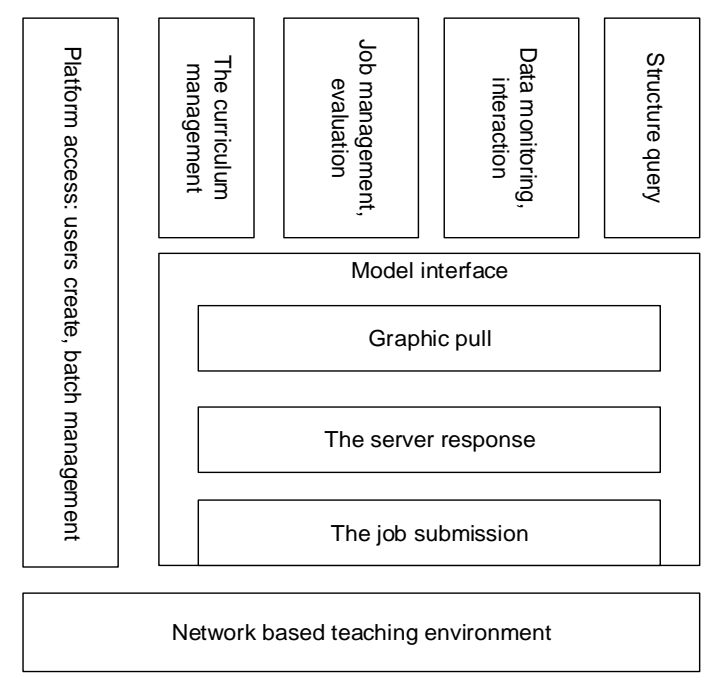

Fig.2. Platform Framework

\section{Establishment of Plantform Environment}

\section{A. Model Selection of Platform Software}

We usually use two software which responsible for teaching communicate platform's establishment and the model of the plug-in support. It provides a data view at the time of containing the actual data. In JGraph products, Mxgraph is a graphical component which provides interface with web integrated. The client requires a Web server to provide the required documents, it can also run on the local file system. The background can be used to support the integrated of existing server language, so it is very friendly to the development.

In consideration of the analysis of the component of model, the platform must also meet the course assignments, including task management modular and data monitor and interact modular. At the same time, the development of language of model plug-in and learning platform need to keep compatibility, so finally we select the current market popular teaching platform software Moodle to meet the needs of establishment of WEB visual modeling and learning platform.

\section{B. Moodle Learning Platformand Mxgraph Graphical Training}

Moodle[3] is an open-source Course Management System (CMS), also known as the Learning Management System (LMS) or Virtual Learning Environment (VLE), which has simple and elegant interface, so users can adjust interface and content according to the requirement at any time. The Moodle platform is a dynamic website program by the PHP scripting language, it is configured of the MySQL database supported by PHP, and it could be used to storage the data and file generated by the Moodle interface.

The creation of graphics is by Mxgraph, which includes all the web[2] development language. Mxgraph client is a graphical component, and it can provide a interface of web integrated. Mxgraph has the architecture and graphics components of MVC, which has the same rules of JGraphthe, and it can be applied in a number of development. The client can realize localized operation, also is convenient to users to develop project alone.

\section{The Key Technology of Visualization Platform}

In the visual modeling training platform development process, the internal structure of the Moodle must be carried out to understand the, including the database and the module of data link. For example, the user who select the corresponding courses in the Moodle will enter into the corresponding modeling interface, and the operation is fed back to the establishment of the database, which also marks the link between two software. To achieve the overall visual programming training platform, it must be associated with Mxgraph and Moodle. Relationship in the user operation with reference to FIG. 2.

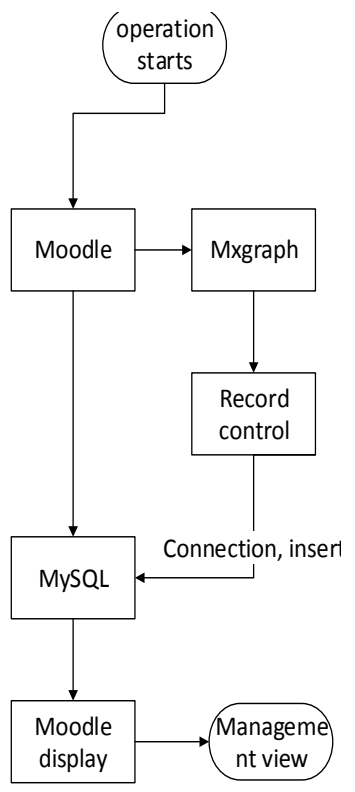

Fig.2. flow chart of software operation platform

\section{A. Graphical Development Training Page in the Mxgraph}

In the Mxgraph, The menu bar and feature-rich tool make creating and editing simple graphics. However, the official demo given too much to call back url and Java code, it's not easy to develop in the front page to connect Moodle. Written by JavaScript and built in the Tomcat server environment. The development of requirements is to refer to the corresponding component, the front page of the project Mxgraph should be met:

1) Drag the icon to create a user model. There are mobile location, modify the size of the function.

2) The above steps can be recorded in real time by the back-end database records. For the above requirements, the project on the local server for Mxgraph develop new homepage.

In response to these requirements, the extraction of the core code of internal mxClient. Js can create a model. Then, Simple graphical configuration pull function that meets the basic requirements. To create a model of the type, according to 
the different needs of different courses, it can be added in the model gallery.

\section{B. Monitoring Training Records by Moodle}

Moodle course modules not only provide support, this project requires the user has a monitoring operation on, that is user real-time monitoring and recording procedures. Monitoring records shall be, user adds a type of block diagram, or the user modifies a block diagram. User visualization and modeling in Mxgraph interface has been developed, in order for managers to understand their structure and thinking to create.

With JavaScript, we can reconstruct the entire Mxgraph client to add some function, including adding, removing, changing or rearranging items on the page. When we want to change any module on the page. JavaScript needs access to the entrance of all the elements in the HTML document, which entrance along with the methods and properties to add, move, change or remove the HTML element, Is obtained through the document object model(DOM).

\section{Structure Analysis of Moodle and Mxgraph and their Interaction}

The first two has completed the creation of the Mxgraph model and the operation of the user record creation process. Then We need to establish the model to transfer to Moodle. And the database recording operation is also uploaded to the result. So what we need to achieve is data exchange between Mxgraph and Moodle. But the data in the Moodle will display where the front in the corresponding position has becoming the key of the platform's development.

The specific steps are provided by the user clicks on the link after elective operation. Table assign submission in the corresponding Mysql in the formation generate the corresponding value. Then the results of MVC model corresponding to the records generate in the online text field in the assign submission online text. At the same time, IP, time to submit homework and so on will be recorded. This text will be submitted in the Moodle view display operation.

The technical scheme is able to complete the relationship and coordination between the two software, presents a complete WEB visualization programming training platform in the server set up after the completion.

\section{Moodle Operation Modular Management}

Easy to operate, to complete the development of the technology of Moodle and Mxgraph after the details of the treatment in the actual operation, the front end service platform forms a teaching system.

Managers can create courses in Moodle, and publish the work that can be placed in the Mxgraph link. The information can be accepted by the users who select corresponding course. After the user selection in the MySQL assign submission database automatically appear in a record, recording the submission user ID and field operation number corresponding to the submission field in assign submission online text with the serial number. Finally, managers can give the score in the submitted after the operation. The score will be saved in the assign grades data sheet.

So far, all the function modules of experimental teaching platform of visual modeling is the development and application. It is not only support the modeling of all kinds of teaching model, but also set up the framework of the platform of information processing, which is used for the analysis of the job submission. Managers judge from the record whether the user can independently accomplish the work directly or theft. It can be in the operation record inquiry knowing, which exceeds the completeness of traditional teaching that can have in the practical application.

\section{Summary}

The visualization platform for educational institutions or small laboratory provides a practical digital teaching, scientific research, management and service network platform. In addition, through the realization of the environment (including equipment, etc.), resources to the application (including teaching, learning, management, service and office), it construct a digital space based on the traditional campus. In the dimension of time and space to expand the real campus the traditional campus, the platform finally realizes the educational process information, so as to improve the management level and efficiency of the aim of education.

\section{Acknowledgments}

The authors gratefully acknowledge financial support from the Practices Education System Improvement Project of Wuhan University of Technology.

\section{References}

[1] Zhiyuan Yuan, Xinqi Zheng, Lina Lv et al.. From design to digital model: A quantitative analysis approach to Garden Cities theory. Ecological Modelling, 2014, 289.

[2] Kartik Kandadai Agaram. Prefetch mechanisms by application memory access pattern. The University of Texas at Austin, 2007.

[3] Zoran Vručinić, Aleksandar Janković, Milan Miladinović et al.. EVALUATION OF CLINICAL DIAGNOSIS WITH "STORE AND FORWARD" TELEDERMATOLOGY. Acta Medica Medianae, 2010, 49(4).

[4] Chris Brunsdon, Lex Comber. Assessing the changing flowering date of the common lilac in North America: a random coefficient model approach. GeoInformatica, 2012, 16(4).

[5] Qian Liu. Securing telehealth applications in a Web-based e-Health portal. Concordia University (Canada), 2008. 